\title{
NURSUSTOOLKIT: UN RECURSO DE ENSEÑANZA- APRENDIZAJE PARA LA SOSTENIBILIDAD EN ENFERMERÍA
}

\author{
NURSUSTOOLKIT: UM RECURSO DE ENSINO-APRENDIZAGEM PARA A \\ SUSTENTABILIDADE NA ENFERMAGEM \\ NURSUSTOOLKIT: A TEACHING AND LEARNING RESOURCE FOR SUSTAINABILITY IN \\ NURSING
}

María Luisa Grande Gascón (mlgrande@ujaen.es)*

Carmen Álvarez Nieto (calvarez@ujaen.es)*

Manuel Linares Abad (mlinares@ujaen.es)*

Isabel María López Medina (imlopez@ujaen.es)*

Gema Parra Anguita (gparra@ujaen.es)*

Cristina Álvarez García**

\section{RESUMEN}

Los profesionales de enfermería están escasamente preparados para hacer frente a los cambios medioambientales y al grave impacto que tienen sobre la salud de la población. También existe un déficit importante en la gestión sostenible de los centros sanitarios donde se generan gran cantidad de residuos. El proyecto que presentamos en este trabajo denominado: NurSusTOOLKIT: A Teaching and Learning Resource for Sustainability in Nursing está financiado en la convocatoria europea Erasmus+ y pretende dar respuesta a esta situación. Se plantea como objetivo mejorar la formación y adquisición de competencias en sostenibilidad medioambiental en los estudios de enfermería, mediante el desarrollo de un sistema de enseñanza-aprendizaje basado en la adopción de enfoques innovadores y la difusión de buenas prácticas a través del uso estratégico de las tecnologías de la información. En el proyecto, que está implementándose en la actualidad, participan investigadores de cuatro universidades europeas y uno de los resultados previstos es el desarrollo de una web de aprendizaje electrónico como recurso de acceso libre con materiales de enseñanza y aprendizaje para mejorar las competencias sobre sostenibilidad en enfermería.

Palabras-llave: Sostenibilidad, cambio climático, Grado en enfermería, salud.

\section{RESUMO}

Os profissionais da enfermagem estão pobremente preparados para enfrentar as mudanças meio ambientais e o grave impacto que essas têm sobre a saúde da população. Também existe um déficit importante na gestão sustentável dos centros de saúde onde se geram grandes quantidades de resíduos. O projeto que apresentamos neste trabalho denominado: NurSusTOOLKIT: Um Ensino e Aprendizagem de Recursos para Sustentabilidade em Enfermagem, financiado pela chamada europeia Erasmus+, pretende dar uma resposta a esta situação. Surge como objetivo melhorar a formação e 


\title{
Egitania \\ s c i e $\Omega$ c i a
}

aquisição de conhecimento na sustentabilidade meio ambiental nos estudos de enfermagem através do desenvolvimento de um sistema de ensino-aprendizagem com base na adoção de abordagens inovadoras e a difusão de boas práticas através do uso estratégico das tecnologias da informação. Neste projeto, que está sendo implementado na atualidade, participam pesquisadores de quatro universidades europeias e um dos resultados planejado é a criação de uma plataforma de aprendizagem eletrônica como recurso de livre acesso com material de ensino e aprendizagem para melhorar o conhecimento da enfermagem sobre a sustentabilidade.

Palavras-chave: sustentabilidade, mudança climática, Licenciatura em enfermagem, saúde.

\begin{abstract}
Nurses are hardly prepared to deal with environmental changes and with the serious impact that they have on the health of the population. There is also an important deficit in the sustainable management of sanitation centers due to the vast amount of waste that is generated. The project presented here is theNurSusTOOLKIT - A Teaching and Learning Resource for Sustainability in Nursing, funded by the European call Erasmus+, which aims to respond to this situation. The main objective is the improvement of training and the acquisition of competences for nursing studies through environmental sustainability and the development of a teaching and learning system based on the acquisition of innovative approaches and on the disseminations of good practices through the strategic use of information technologies. In this project, which is currently being implemented, researchers at four European universities aim to development of a web page for electronic learning as an open source of teaching and learning materials to improve competences in sustainability in nursing.
\end{abstract}

Keywords: Sustainability, climate change, Nursing degree, health.

\author{
* Profesores Titulares de la Universidad de Jaén \\ ** Alumna de la Universidad de Jaén
}

Submitted: 23th June 2015

Accepted: 17th November 2015 


\section{Eqgitania}

s c i e $\Omega$ c i a

\section{INTRODUCCIÓN}

Si partimos de la premisa de que el cambio climático supone una amenaza para la salud, los servicios sanitarios deben de estar preparados para hacer frente a las consecuencias que sobre la salud tiene el cambio climático, garantizando la sostenibilidad y la prestación de servicios. Los cambios ambientales como las olas de calor, el aumento del nivel del mar y el aumento de la sequía incrementan los problemas de salud existentes y la aparición de otros nuevos. Las olas de calor agravan las enfermedades cardiovasculares, renales y respiratorias, la diabetes y los trastornos del sistema nervioso. El aumento del ozono a nivel del suelo produce asma, enfermedades respiratorias y cardiacas, como arritmias; mientras que su disminución en capas altas, incide en una mayor presencia de cataratas, cáncer de piel, aumento de vectores transmisores de enfermedades como la malaria, etc. Las catástrofes ocasionadas por la lluvia como inundaciones, o las sequias también producen problemas graves de salud. El aumento del nivel de mar produce salinidad en aguas superficiales, y pone en peligro el agua potable disponible y la producción agrícola (Sayre et al., 2010).

Por lo tanto, el desarrollo sostenible es un concepto vital para la salud debido a las consecuencias que el aumento de las emisiones de dióxido de carbono (CO2), el uso de materiales nocivos y la producción de grandes cantidades de residuos tiene sobre la salud. Los contaminantes químicos, combustibles fósiles y las radiaciones generan la contaminación del agua y del aire y son graves problemas en nuestra sociedad actual. Para hacer frente a los mismos es necesaria una reflexión crítica sobre la realidad social y un cambio de actitudes y creencias desde una visión ecológica integral (Svaldi et al., 2013).

La asistencia sanitaria puede verse comprometida y en última instancia la salud pública, como consecuencia de los daños a la capacidad de las generaciones futuras para satisfacer sus necesidades básicas. Por lo tanto la gestión adecuada de los residuos, la reducción del uso de la energía y el incremento de la eficiencia en el uso de los recursos son elementos vitales para conseguir que los sistemas sanitarios sean más sostenibles. Por destacar un dato importante, se calcula que en la Unión Europea (UE), el sector de la salud produce aproximadamente un 5\% de las emisiones totales de CO2 (KPMG, 2012).

La mejora de la eficiencia energética y de recursos, las políticas de contratación y gestión de residuos son vitales para que la sanidad sea más sostenible. El reciente informe del Grupo Intergubernamental de Expertos sobre el Cambio Climático hace hincapié en el riesgo que tiene el cambio climático para la salud humana; todos los países se encuentran en riesgo y es necesario reducir los impactos en el clima. Diferentes investigaciones proponen un mayor liderazgo de la UE en las políticas relacionadas con el cambio climático que priorice la salud de las personas. La iniciativa emblemática de Europa 2020 subraya la necesidad de una transición hacia el uso de los recursos naturales de manera más eficiente, mejorando así la gestión de los recursos energéticos y la eficiencia de los costes en la atención sanitaria, porque está demostrado que de esta manera se logra promover una buena salud, se reducen los costes sanitarios y se posibilita un envejecimiento saludable (Holland, 2010). 


\section{Egitania}

$s$ c i e $\Omega$ i a

\section{ESTADO DE LA CUESTIÓN}

Si un medio ambiente sano es importante para una vida sana, una pregunta que surge es como afectan las actividades del personal sanitario, y más especificamente las de enfermería, al medio ambiente. Tal y como sostienen Anaker and Elf (2014), la conciencia ambiental en enfermería es muy importante y es necesario un incremento de la sensibilización en temas medioambientales (López Fernández et al., 2009).

Partiendo de esta premisa, es fundamental una gestión medioambiental en los centros sanitarios por parte del personal de enfermería, pues es clave para la prevención de la contaminación mediante el uso de materiales ambientalmente seguros. Mediante una adecuada formación ambiental, las enfermeras deben conocer cuáles son los cambios necesarios que hay que introducir en la práctica de enfermería a fin de mantener una relación sostenible con el medio ambiente. Y para ello el alumnado de enfermería debe adquirir conocimientos y desarrollar competencias en estos aspectos y así paliar las significativas consecuencias que está ocasionando el cambio climático (López Fernández, 2009; Stavrianopoulos et al., 2010).

Las enfermeras son agentes de cambio que tienen la capacidad necesaria para promover la salud y controlar el uso de los recursos sanitarios. Por tanto, mejorar el nivel de conocimientos y habilidades sobre sostenibilidad y los efectos del cambio climático en la formación enfermera eliminará esta brecha educativa y posibilitará un cambio de actitudes y modos de actuación en su labor diaria, que redundará en última instancia en la mejora de la salud de la población (Richardson et al., 2014).

A nivel general se consiguen grandes beneficios con el incremento de las iniciativas de concienciación ciudadana sobre el medio ambiente en los medios de comunicación, que difunden estrategias que inciden en los comportamientos para conseguir una relación sostenible con el medio ambiente. También las conductas individuales, que pueden servir de ejemplo a los pacientes y a otros profesionales, pueden favorecer la preservación del medio ambiente. En todas estas iniciativas pueden participar los profesionales de enfermería, siempre y cuando se desarrollen en el marco de politicas adecuadas orientadas a la sostenibilidad ambiental (Goodman, 2011; Camponogara et al., 2014; Butterfield et al., 2014).

Por este motivo es importante, expandir las estrategias educativas y de investigación para hacer frente a los peligros globales, que cada vez son más evidentes, entendiendo la salud de una manera integral dentro de un contexto físico, social, económico y político (Johnston et al., 2005; Leffers y Mitchell, 2011). También se debe tener en cuenta aquellas cuestiones globales que afectan de manera importante a la salud como la pobreza, la estabilidad mundial, la igualdad de género, la libertad, la violencia y la guerra. Todas estas circunstancias además tienen incidencia de manera directa o indirecta en la sostenibilidad del medio ambiente, en un contexto mundial caracterizado por el uso masivo de las tecnologías de la información y la comunicación y donde el contexto político e institucional juega un papel fundamental. Para ello se deben tomar 


\section{Egitania}

s c i e $\Omega$ c i a

decisiones a largo plazo, teniendo en cuenta las circunstancias actuales, pensando así en escenarios futuros posibles, plausibles, probables y preferibles (Johnston et al., 2005).

Para lograr esto es necesario desarrollar programas de sostenibilidad, que puedan ser modificados a través del tiempo, para que encajen en los procedimientos y la ideología de la organización, y que tengan en cuenta los beneficios para el personal y los pacientes. Es importante la participación de otras organizaciones interesadas que pueden brindar apoyo, y así fomentar el liderazgo del personal sanitario y la participación. Un requisito fundamental es que la financiación y la planificación en cualquier ámbito deben ser sostenibles (Clarke y MarksMaran, 2014). En definitiva el personal sanitario y los responsables políticos deben prepararse para el cambio climático mediante la formación y la adaptación desde un enfoque multidisciplinar necesario para hacer frente al mismo y a la repercusión que tiene sobre la salud individual y colectiva (Sayre et al., 2010). Hay que ser conscientes de que las tendencias demográficas, la tecnología, el clima y la disponibilidad de recursos pueden afectar nuestra capacidad de proporcionar atención sanitaria en el futuro (Thompson et al., 2010).

Los sistemas sanitarios deben implementar prácticas sostenibles en torno a la generación de residuos y el uso de la energía, que deben tener como soporte políticas públicas medioambientales y políticas de gestión integradas en estructuras e institucionalizadas en los procesos corporativos, teniendo en cuenta que los problemas están interconectados en diferentes niveles (políticos, económicos, sociales, culturales, ) y que por lo tanto no deben ser abordados de manera aislada (Goodman, 2011; Sari y Camponogara, 2014; Butterfield et al., 2014).

Para ello es fundamental una financiación suficiente y el apoyo de los sistemas nacionales de salud de cada país. Las iniciativas gubernamentales y por parte de los profesionales deben incluir filosofías, estándares y objetivos vinculados a la sostenibilidad medioambiental (López Fernández, 2009). Hay que buscar soluciones energéticas sostenibles y para ello son necesarias políticas de reducción de las emisiones de carbono tanto desde los gobiernos nacionales como desde los organismos internacionales (Richardson et al., 2014; Stavrianopoulos et al., 2010).

Desde el ámbito académico en los planes de estudio de enfermería es fundamental la inclusión de competencias y contenidos sobre sostenibilidad medioambiental, que incida en la reducción de los contaminantes, desde una visión crítica y reflexiva. De tal manera que la responsabilidad social y la idea de preservación del planeta estén presentes en la formación de enfermería. Lo que se debe conseguir son conocimientos sólidos y una reflexión crítica, teniendo en cuenta la adquisición de habilidades cognitivas, afectivas y conductuales. (Goodman, 2011; Barna, 2012; Lopes Monteiro et al., 2010; Camponogara et al., 2012).

Así, las enfermeras podrían realizar medidas de educación para la salud teniendo en cuenta estos planteamientos, desde un enfoque multidisciplinar que incluya aspectos relacionados con la economía, política, diseño, filosofía, medio ambiente, literatura, etc. El objetivo debe ser el 


\section{Egitania}

s c i e $\Omega$ c i a

bienestar individual y comunitario y debe ir más allá del modelo biomédico; el enfoque utilizado debe estar en consonancia con una visión holística de la salud (Goodman, 2011).

Las investigaciones realizadas demuestran que las enfermeras no están preparadas, sólo realizan en torno a la mitad de las tareas teniendo en cuenta planteamientos medioambientales. Actualmente existe una demanda creciente, desde diferentes ámbitos, de incluir los problemas de sostenibilidad y cambio climático en el currículum de enfermería (Middleton et al., 2014; Sterling et al., 2005; Goodman, 2011).

Según señala Kirk (2002), "Ios estudiantes de enfermería están siendo mal preparados para entender las conexiones entre el cambio climático, la sostenibilidad y la salud". Sin embargo, resulta fundamental que los profesionales de la salud conozcan los conceptos de sostenibilidad y cambio climático, y la relación que tienen con la salud y el bienestar de las personas (Goodman, 2011; Goodman et al., 2010). Precisamente uno de los objetivos de la educación medioambiental es conceptualizar la salud y la enfermedad teniendo en cuenta los aspectos biológicos, psicológicos, sociales y ecológicos. El conocimiento de todos los elementos que configuran la salud sostenible puede ayudar al personal de enfermería a mejorar su trabajo, y de esta manera se puede mejorar la salud de las personas a las que presta atención sanitaria (Lopes Monteiro et al., 2010; Barna et al., 2012).

En este sentido, es necesaria una formación específica para tener profesionales capacitados que en su desempeño profesional tengan en cuenta la sostenibilidad medioambiental. Dicha formación les ayudará en el desarrollo de iniciativas que tenga incidencia política, porque hay que tener en cuenta que los avances en este sentido solo se logran con el apoyo institucional y de las organizaciones profesionales. Además es importante el desarrollo de una especialización de enfermería que transcienda la formación de grado y se extienda a la formación de posgrado y a la formación continuada, incluyendo todo lo relacionado con la investigación que debe ir en paralelo a dicha formación. (Andrews, 2009; Richardson et a.,I 2014; Goodman, 2013; Dunphy, 2014).

Las enfermeras para proporcionar cuidados integrales deben conocer la contribución que ha tenido la actividad humana en los cambios ambientales globales, como el cambio climático, la pérdida de biodiversidad y el agotamiento de recursos. Además, deben comprender cómo las repercusiones sanitarias del cambio ambiental tienen una distribución desigual dentro y entre las poblaciones, y la disparidad existente entre los más responsables y los más afectados por el cambio. En todo este planteamiento, es importante el abordaje de las cuestiones éticas que se producen en la asignación de recursos entre los pacientes de manera individual y colectivo

y la protección del medio ambiente, de la que la salud de la comunidad en general depende (Thompson et al., 2010; Andrews, 2009).

Otro aspecto importante es el papel de enfermería en la gestión de los residuos y desechos sanitarios de los servicios de salud que debe pasar por un proceso planificado que incluye aspectos relacionados con la generación, minimización, segregación, reciclaje, envasado, almacenamiento, recolección, tratamiento y disposición final. El objetivo que tiene que 


\section{Eqgitania}

$s$ c i e $\Omega$ c i a

conseguirse es la conservación de recursos, reducción y eliminación de residuos biológicos, físicos y agentes químicos que suponga un riesgo para la población (Anaker y Elf, 2014; Richardson et al. 2014; Butterfield et al., 2014; Sayre et al., 2010; Goodman y East, 2014; Goodman, 2013; Campbell, 2008; Correa et al., 2007).

El conocimiento de la gestión adecuada de residuos es un tema muy relevante así como su eliminación, debido a la escasez de recursos y a la necesidad de un adecuado uso de los mismos. De ahí la importancia de identificar los limitados recursos globales utilizados en la atención de enfermería, considerando los recursos disponibles, incluidos los financieros, bienes y servicios, y cómo utilizarlos mejor para garantizar una atención de enfermería equitativa para toda la población mediante la elaboración e implementación de estrategias que minimicen la producción de los residuos sanitarios en la práctica de enfermería (Sendall et al., 2013; Richardson et al., 2014; Sayre et al., 2010; Richardson et al., 2014a; Goodman, 2013).

El objetivo final es lograr centros sanitarios sostenibles, y para ello se necesita una gestión ecológica de los centros basada en el uso de materiales, tecnologías y productos ecológicamente seguros. Para reordenar las prácticas del personal sanitario relacionadas con la generación de residuos, la eliminación de residuos industriales y el consumo de recursos ambientales, es importante la puesta en marcha de planes de gestión ambiental, y la formación de los profesionales en sostenibilidad. En definitiva hay que cambiar el enfoque de los centros sanitarios desde uno meramente mecanicistas orientados biomédicamente, hacia un enfoque más integral y holístico donde los aspectos medioambientales estén presentes en todos los procesos. (López Fernández, 2009; Camponogara et al, 2012; Campbell, 2008; Butterfield et al, 2014; Sari y Camponogara, 2014).

Por lo tanto, la enseñanza y el aprendizaje medioambiental deben estar presentes en la formación universitaria de grado y posgrado mediante la inclusión de competencias específica en los planes de estudios sobre sostenibilidad medioambiental, desde un punto de vista teórico y empírico que enmarque la práctica global de los profesionales de enfermería (Sterling et al., 2005; Goodman, 2011; Barna et al., 2012; Butterfield et al., 2014; Johnston et al., 2005; Leffers y Mitchell, 2011; Galiana y Gascón, 2004).

Deben ser planes de estudios basados, además de en conceptos de sostenibilidad ambiental, en temas de justicia global y uso de la tecnología y sus límites (Johnston et al., 2005). Además los planes de estudios deben ser diseñados para aumentar la capacidad educativa y las prácticas académicas relacionadas con el medio ambiente, que integren otros aspectos relacionados como la salud pública, las desigualdades, la pobreza, la seguridad alimentaria y las enfermedades infecciosas, sin olvidar las habilidades de gestión y liderazgo. Johnston et al. (2005) y McPhee (2009) proponen que estos temas deberían abordarse en el último curso del Grado como culminación del programa formativo de enfermería. En todo caso existe un gran consenso en que lo más importante es reforzar el plan de estudios de enfermería teniendo en cuenta los cambios socio-ambientales, y la sostenibilidad y que aborden aspectos tales como la promoción de la salud ecológica, la justicia social, la igualdad económica, los derechos 


\section{Eqgitania}

s c i e $\Omega$ c i a

humanos, el desarrollo sostenible y que se base en la idea de conseguir un mundo mejor (Sendall et al., 2013; Lopes Monteiro et al., 2010; Sari y Camponogara, 2014; Middleton et al., 2014; Stavrianopoulos et al., 2010; Richardson et al., 2014).

En definitiva el objetivo es formar a las enfermeras para que proporcionen cuidados de salud sostenibles. Pero esto sólo se consigue mediante la implicación de las universidades para dar respuesta al cambio climático global y el agotamiento de los recursos. Incluso la formación de pregrado podría incluir contenidos básicos en sostenibilidad a nivel introductorio para que el alumnado de enfermería tuviera una base y estuviera sensibilizado ante estas cuestiones (Middleton et al., 2014; Butterfield, et al., 2014).

Con este planteamiento varias universidades europeas planteamos un proyecto, que está siendo implementado en la actualidad, con el objetivo conseguir una formación de enfermería más acorde con los retos medioambientales que tendrán que asumir los futuros profesionales de enfermería.

\section{DESCRIPCIÓN DEL PROYECTO}

El proyecto denominado NurSusTOOLKIT: A Teaching and Learning Resource for Sustainability in Nursing está financiado por la convocatoria Erasmus + dentro de la línea de cooperación e innovación en buenas prácticas. Tiene una duración de tres años, siendo la fecha de inicio en 2014 y finalizando en 2017.

Participan cuatro universidades europeas: la Universidad de Plymouth (Reino Unido), Universidad de Jaén (España), Universidad de Esslingen (Alemania) y Universidad de Maastricht (Holanda). La Universidad de Plymouth es la que lidera y coordina el proyecto.

Las investigaciones realizadas no han podido identificar ningún proyecto similar centrado en la enfermería y la sostenibilidad. El proyecto se suma a iniciativas más amplias sobre la educación para un desarrollo sostenible y proporciona una oportunidad para elaborar nuevos recursos de enseñanza y aprendizaje con el fin de abordar algunos de los problemas más difíciles a los que se enfrenta la atención sanitaria en el siglo XXl relacionados con el cambio climático y el agotamiento de los recursos naturales. El eje sobre el que gira el proyecto es el desarrollo de una plataforma digital de recursos vinculados a competencias clave con lo que se conseguiría: facilitar el acceso a la información actualmente disponible en este ámbito; el desarrollo de materiales educativos adicionales, que promueven la interacción y la participación; el diseño de enfoques pedagógicos sostenibles que permitan a los educadores de enfermería de toda Europa integrar la sostenibilidad en el currículum de enfermería de una manera innovadora; y proporcionar oportunidades de aprendizaje a través de la formación profesional continua.

El proyecto se basa en el trabajo realizado por las universidades participantes lo que proporciona un valor añadido a nivel de la Unión Europea a través de la colaboración de universidades de

diferentes países europeos. Por ejemplo, la universidad que coordina el proyecto ha 
desarrollado sesiones formativas para el desarrollo de habilidades basadas en la sostenibilidad medioambiental y que ha tenido impactos evidentes sobre los conocimientos y las actitudes del alumnado universitario. Por este motivo se ha incluido en el plan de estudios de enfermería en la Universidad de Plymouth. La Universidad de Maastricht ha utilizado el Aprendizaje Basado en Problemas para enseñar temas de sostenibilidad en diferentes disciplinas. Así mismo, se ha realizado una encuesta reciente por las universidades participantes sobre las actitudes del alumnado hacia el cambio climático y la inclusión de la sostenibilidad en el plan de estudios de enfermería, siendo una de las conclusiones que estos temas se perciben como relevantes para la práctica clínica.

Este proyecto tiene un carácter innovador en varios sentidos: desarrollará y categorizará publicaciones y recursos disponibles basados en la evidencia, y que desarrollen un marco para la formación en sostenibilidad que sea incluido en los estudios de enfermería; desarrollará y probará nuevos materiales de enseñanza y aprendizaje basados en las tecnologías de la información y la comunicación para apoyar la formación en sostenibilidad y la adquisición de competencias en este ámbito; integrará pedagogías de sostenibilidad; desarrollará diferentes aspectos del plan de estudios con implicaciones prácticas para la enfermería y donde los recursos didácticos pueden apoyar la adquisición de competencias; creará oportunidades de enseñanza y de aprendizaje para facilitar el debate entre el alumnado acerca de temas clave en materia de salud como el cambio climático y el agotamiento de los recursos naturales; desarrollará competencias de sostenibilidad que apoyen el marco de la formación en sostenibilidad. El principal resultado será una herramienta denominada NurSusTOOLKIT basada en una página web de libre acceso para los estudiantes de enfermería, profesionales y docentes que posibilitará el trabajo en colaboración. De esta manera se asegurará que un gran número de recursos estarán disponibles para cubrir el déficit actual, teniendo en cuenta las diferencias culturales.

Los destinatarios de los resultados de este proyecto son: docentes y alumnado de enfermería, profesionales de enfermería y responsables políticos y administrativos implicados en el diseño del currículum de enfermería.

\section{OBJETIVOS}

El objetivo del proyecto es mejorar la formación y adquisición de competencias en sostenibilidad medioambiental en los estudios de enfermería, mediante el desarrollo de un sistema de enseñanza-aprendizaje basado en la adopción de enfoques innovadores y la difusión de buenas prácticas a través del uso estratégico de las tecnologías de la información.

Como objetivos específicos se plantean los siguientes:

- Desarrollar un recurso basado en la evidencia científica sobre sostenibilidad, cambio climático y salud. 


\section{Eqgitania \\ $\mathrm{s}$ c i e $\Omega$ c i a}

- Identificar ejemplos de buenas prácticas y oportunidades para la integración de la sostenibilidad en los planes de estudios de enfermería.

- Desarrollar y acordar un modelo de formación en sostenibilidad vinculado a perfiles profesionales dentro de la enfermería.

- Diseñar un programa de educación con e-módulos comunes y una variedad de estrategias y materiales de enseñanza y aprendizaje.

- Aplicar enfoques innovadores y facilitar el reconocimiento y certificación de habilidades y competencias en materia de sostenibilidad.

- Crear materiales de enseñanza on line (NurSusTOOLKIT) vinculados a los créditos ECTS.

- Difundir los resultados y promover el uso de NurSusTOOLKIT.

\section{RESULTADOS PREVISTOS}

\section{Resultado 1:}

Elaboración de un recurso basado en la evidencia sobre sostenibilidad, cambio climático y salud. Para ello se realizará una revisión sistemática de investigaciones publicadas y de recursos on-line con el fin de identificar los recursos y enfoques pedagógicos en relación con el desarrollo y la utilización de materiales docentes en estos temas

Con esta información se elaborará un artículo basado en la revisión realizada que será enviado para su publicación en una revista internacional de enfermería con el fin de que tenga una amplia difusión, elevando así el perfil del proyecto. En el documento se utilizará la evidencia encontrada para resumir las principales cuestiones de enfermería en relación a la sostenibilidad y la salud. Los recursos identificados y categorizados serán cotejados en formato electrónico de acuerdo a las áreas temáticas y se pondrá a disposición de las instituciones asociadas a NurSusTOOLKIT a través de páginas web.

\section{Resultado 2:}

Realización de un análisis temático de los planes de estudios con el objetivo de identificar oportunidades para incluir la sostenibilidad en los planes de estudios de enfermería en los diferentes países europeos. Esta actividad se complementará con el desarrollo de un Estudio Delphi. De esta manera se conseguirá una visión integral acerca de las competencias sobre sostenibilidad, las oportunidades curriculares y los obstáculos para la formación en enfermería. Con esta información se elaborará un documento donde se destaquen las oportunidades de integrar la sostenibilidad en los planes de estudio de enfermería que será difundido en foros científicos y profesionales. 


\section{Egitania}

$s$ c i e $\Omega$ c i a

El estudio Delphi posibilitará un proceso participativo más amplio y permitirá la identificación de cuestiones clave y buenas prácticas integradas en los planes de estudio de enfermería. Algunos de las cuestiones que se incluirán en el Delphi son: el significado de sostenibilidad en el cuidado de la salud, la importancia de la formación en sostenibilidad en el currículum enfermero y las barreras percibidas para la integración de la sostenibilidad en la formación de enfermería. Para el estudio Delphi se contará con docentes universitarios, gestores de enfermería e investigadores que puedan aportar desde su experiencia información relevante sobre las cuestiones planteadas.

\section{Resultado 3:}

Desarrollo del marco de conocimientos y competencias en sostenibilidad que constituirán la base para el desarrollo de materiales educativos y la creación del NurSusTOOLKIT. Se identificarán pedagogías que puedan apoyar el proceso completo de aprendizaje, diseñando un rango que aseguren la relevancia para el desarrollo profesional continúo. Tanto la selección de materiales de aprendizaje como las estrategias didácticas apropiadas e innovadoras estarán basadas en la evidencia científica.

El marco incluye contenidos relevantes en sostenibilidad, competencias centrales de sostenibilidad para enfermería y pedagogías en las que se base todo el proceso de aprendizaje. Los contenidos previstos incluyen áreas como: atención sanitaria a los problemas de salud derivados del cambio climático, teniendo en cuenta los determinantes de salud; prevención de enfermedades basada en los beneficios derivados de comportamientos y formas de vida más sostenibles; uso y manejo de recursos naturales, incluyendo la gestión sostenible de los residuos; y liderazgo y gestión de la complejidad y la incertidumbre. El contenido se dividirá en cursos y módulos en los que se vincularán la teoría y la práctica, y las estrategias pedagógicas se diseñarán haciendo énfasis en enfoques participativos.

\section{Resultado 4:}

Ensayo y evaluación de los materiales de aprendizaje en el contexto del currículo de enfermería y desarrollo de una estrategia de contenidos para el NurSusTOOLKIT. Los materiales se ensayarán y evaluaran con estudiantes de enfermería, docentes y profesionales a través de cuestionarios específicos para cada uno de los grupos participantes. Con esta actividad se pretende evaluar los materiales de enseñanza y aprendizaje diseñados para el NurSusTOOLKIT. Todas las universidades integradas en el proyecto participaran en la evaluación, así como otros grupos de interés con los que se tengan vínculos.

\section{Resultado 5:}

Desarrollo de una web de aprendizaje electrónico denominada NurSusTOOLKIT como un recurso de acceso libre con materiales de enseñanza y aprendizaje para mejorar las 


\section{Eqitania}

s c i e $\Omega$ c i a

competencias sobre sostenibilidad en enfermería que estará disponible en varios idiomas. Este es el principal resultado del proyecto e integrará lo desarrollado en las fases previas.

El NurSusTOOLKIT tendrá tres componentes:

- Un banco de recursos clasificados de acuerdo a la materia de que se trate en cada caso.

- Contenidos nuevos de enseñanza y aprendizaje desarrollados en forma de cursos y módulos organizados por temas y apoyados por pedagogías didácticas y participativas, ligadas a competencias y a los créditos ECTS.

- Guía de uso del NurSusTOOLKIT en varios idiomas.

\section{Resultado 6:}

Diseño de un plan para la difusión y explotación del NurSus TOOLKIT a nivel local, regional y europeo, adaptado a los diferentes grupos de destinatarios entre los que se contará con diferentes tipos de organizaciones, tanto nacionales como transnacionales.

El profesorado de enfermería y el alumnado serán los destinatarios principales del NurSus TOOLKIT, y se le darán las instrucciones para su uso. Además otros expertos pueden obtener la información de cómo usar el NurSus TOOLKIT como parte de su desarrollo profesional continuo.

Los responsables de la toma de decisiones en diferentes niveles recibirán información sobre el NurSusTOOLKIT, así como los investigadores y la población en general. Para ello se enviarán boletines (E-newsletters) de noticias cada seis meses, se creará un blog donde los usuarios puedan conocer y debatir sobre experiencias, se publicaran artículos en revistas científicas y se enviarán comunicados a los medios de comunicación.

En relación al impacto previsto del proyecto, se prevé un incremento de la conciencia sobre sostenibilidad y cambio climático y de la importancia de su inclusión en la formación enfermera. De esta manera habrá un aumento de las competencias sobre sostenibilidad en los planes de estudios de enfermería, de las que se beneficiarán en un primer momento más de 2.000 estudiantes por año. Además las universidades participantes se comprometen a promover la sostenibilidad en sus actividades formativas de tal manera que se seguirá trabajando en las actividades diseñadas en este proyecto una vez finalizado.

A nivel europeo se pretende que se conozcan las estrategias para integrar la sostenibilidad en los planes de estudio de enfermería en todos los países de la Unión Europea. A través de enlaces a NurSusTOOLKIT se posibilitará un amplio acceso a la misma desde los sitios web de las entidades interesadas, tanto en el ámbito académico como profesional. Así mismo el NurSusTOOLKIT será diseñado para fomentar modelos de buenas prácticas de las organizaciones sanitarias y educativas que se añadirán a fin de que la información se mantenga actualizada y sea relevante para la práctica profesional. Esto fomentará el compromiso 


\section{Egitania}

$s$ c i e $\Omega$ c i a

permanente con los materiales. Para el alumnado el impacto vendrá por la posibilidad de realizar formación online pues el NurSusTOOLKIT estará ampliamente disponible a través internet, utilizando sitios web asociados, lo que además asegurará que puedan acceder personas de diferentes países y regiones del mundo.

\section{CONCLUSIONES}

Las enfermeras deben conocer las consecuencias del cambio climático en la salud, así como la relación existente entre el medioambiente y la salud. En este sentido deben adquirir habilidades específicas en este campo relacionadas con la sostenibilidad medioambiental y el cambio climático, así como competencias en la gestión sostenible de los centros sanitarios.

Las competencias necesarias para una actuación acorde con el medio ambiente deben adquirirse tanto en la formación de grado como de posgrado, haciéndola extensiva a la formación profesional continuada. Para ello es necesaria más investigación sobre enfermería en materia de salud medioambiental. Así se conseguirá el liderazgo de enfermería en este ámbito, de modo que los profesionales de enfermería serán capaces de hacer frente al impacto del cambio climático en la salud, hacer un uso eficiente de los recursos y abordar la gestión de residuos y desechos que se generan en los centros sanitarios.

Es fundamental que enfermería logre una formación sostenible, ya que los cambios climáticos afectan de forma intrínseca a la salud y las enfermeras debemos proporcionar un cuidado holístico, atendiendo a todas las dimensiones de nuestros pacientes. Como se ha puesto de relieve las enfermeras están escasamente preparadas en materia medioambiental por lo que se hace necesario modificar los planes de estudio, tanto de grado como de posgrado. Son numerosos los expertos que apoyan esta idea y ya hay una amplia gama de propuestas en este sentido, tanto en relación a los contenidos teóricos y prácticos como a los enfoques pedagógicos a utilizar.

Tras la búsqueda de recursos sobre sostenibilidad en enfermería, se puede afirmar que son escasos los específicos de cuidados sostenibles destinados a enfermería, no siendo así para los de salud medioambiental en general. Este tipo de recursos son muy útiles para la formación, ya que son muy versátiles, de ahí la importancia de crear más.

El proyecto que se está implementando pretende dar respuesta a los déficits que la formación enfermera tiene en este ámbito. El diseño de un instrumento como es el NurSusTOOLKIT, tendrá un gran impacto a nivel internacional y posibilitará que se conozcan las estrategias para integrar la sostenibilidad en los planes de estudio de enfermería en todos los países de la Unión Europea. A través de internet se podrá acceder al NurSusTOOLKIT, tanto en el ámbito académico como profesional. Así mismo fomentará modelos de buenas prácticas útiles para las organizaciones sanitarias y educativas. 


\section{Egitania}

s c i e $\Omega$ c i a

\section{AGRADECIMIENTOS}

A Andrea Galloti por sus aportaciones al artículo.

\section{REFERÊNCIAS}

ANAKER, A. Y ElF, M. (2014). SUSTAINABILITY IN NURSING: A CONCEPT ANALYSIS. SCANDINAVIAN JOURNAL OF CARING SCIENCES, $28(2), 381$ 389.

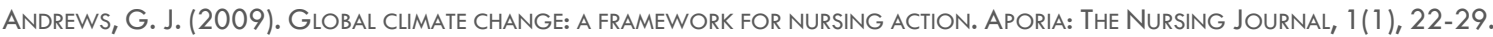

Barna, S., Goodman, B. y Mortimer, F. (2012). The health effects of climate Change: What does a nURSE need to kNOW? NURSE EDUCATION TODAY, 32(7), 765-771.

Butterfield, P., SChenk, E., Eide, P., Hahn, L., Postma, J., Fitzgerald, C., et Al. (2014). IMplementing AACN'S ReCommendations for ENVIRONMENTAL SUSTAINABILITY IN COLLEGES OF NURSING: FROM CONCEPT TO IMPACT. JOURNAL OF PROFESSIONAL NURSING, 30(3), 196-202.

Campbell, S. (2008). Nursing the planet. Primary Health Care, 18(3), $41-48$.

Camponogara, S., Soares, S.G.A., Viero, C.M., Erthal, G., Diaz, P.S., Peres, R.R., et al. (2012). Responsabilidade ambiental na visão de ACADÊMICOS DA ÁreA DA SAÚDE. REVISTA ENFERMAGEM UERJ, 20(1), 39-44.

Clarke, U., Marks-Maran, D. (2014) Nurse leadership in Sustaining programmes of Change. British Journal Of Nursing, $23(4), 219$ 24.

CorRÊA, L.B., LUNARDI, V.L., De CONTO, S.M. (2007). O PROCESSO DE FORMAÇÃo EM SAÚdE: O SABER RESídUOS SÓLIDOS DE SERVIÇOS DE SAÚdE EM VIVÊNCIAS PRÁtICAS. REVISTA BRASILEIRA DE ENFERMAGEM, 60(1), 21-25.

DUNPHY, J. L. (2014). HeAlthCARE PROFESSIONAlS' PERSPECTIVES ON ENVIRONMENTAL SUSTAINABILITY. NURSING ETHICS, 21 (4), 41 4-425.

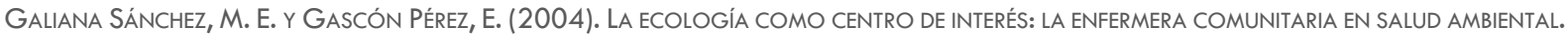
REVISTA ROL DE ENFERMERÍA, 27(1), 21-28.

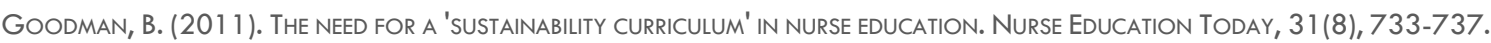

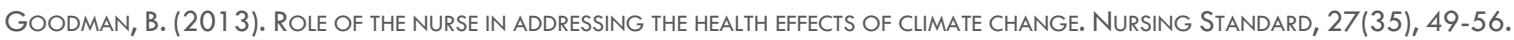

GOODMAN, B. y EAST, L. (2014). THE 'SUSTAINABILITY LENS': A FRAMEWORK FOR NURSE EDUCATION thAT IS 'FIT FOR THE fUtURE'. NURSE EdUCATION TODAY, 34(1), 100-3.

Goodman, B. y Richardson, J. (2010). Climate Change, Sustainability and Health in UK Higher Education: The Challenges for Nursing. En Jones, P., Selby, D. y Sterling, S. (Eds), Sustainability Education Perspectives and Practice Across higher Education. (pp. 109-132). LONDON: EARTHSCAN.

Holland, M. (2010). Acting now for better health: A 30\% Reduction Target for eu Climate Policy (2010). Healthcare Without harm / health and Environment Alliance. Recuperado el 7 de abril, 2015 de http://WWW.enV-health.org/IMG/PdF/1_ACTING_NOW_FOR_A_BETTER_HEALTH_-_A_REDUCTION_TARGET_FOR_EU_CLIMELATE_POLICY.PDF

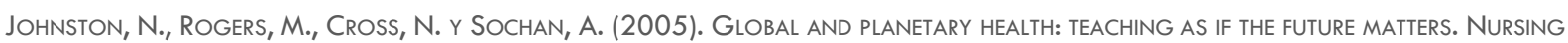
EDUCATION PerspeCtives, 26(3), 152-156.

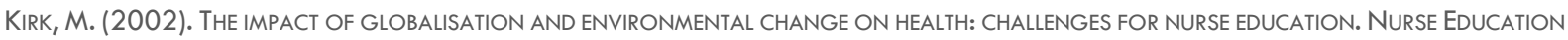
TODAY, 22(1), 60-71 


\section{Egitania \\ $s$ c i e $\Omega$ c i a}

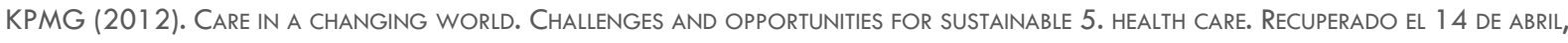
2015 DE HTTP://WWW.KPMG.COM/GLOBAL/EN/ISSUESANDINSIGHTS/ARTICLESPUBLICATIONS/CARE-IN-A-CHANGINGWORLD/DOCUMENTS/CHALLENGES-OPPORTUNITIES-SUSTAINABLE-HEALTHCARE.PDF

Leffers, J. y Mitchell, E. (2011). ConCeptual Model for Partnership and Sustainability in Global Health. Public Health Nursing, 28(1), 91-102.

López Fernández, M.T., Pastor Torres, E., Sánchez Sauco, M.F., Ferris Tortajada, J. y Ortega García, J.A. (2009) Enfermería en SAlud MEDIOAMBIENTAL. EXPERIENCIA EN UNA UNIDAD DE SALUD MEDIOAMBIENTAL PEDIÁtrICA. ENFERMERÍA CLÍNICA., 19(1), 43-47.

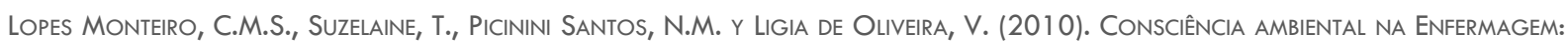
RECONSTRUÍNDO UM MUNDO MELHOR COM A CONTRIBUIÇÃO DOS ESTUDANTES. REFERÊNCIA, III(2), 35-43.

MacPhee, M., WejR, P., Davis, M., Semeniuk, P. y SCARborough, K. (2009). Practice and ACAdemic nurse educators: Finding common GROUND. INTERNATIONAL JOURNAL NURSING EDUCATION SCHOLARSHIP, 6(1), 20 P.

Middleton, L., Howard, A.A., Dohrn, J., Von Zinkernagel, D., Hopson, D.P., Aranda-Naranjo, B., et al. (2014). The Nursing

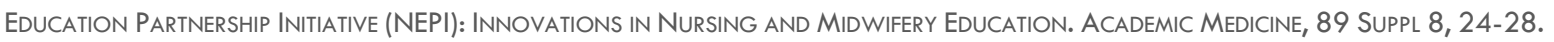

Richardson, J., Grose, .J, DOMAN, M. Y KelSEY, J. (2014). THE USE OF EVIDENCE-INFORMED SUStAINABILITY SCENARIOS IN the NURSING

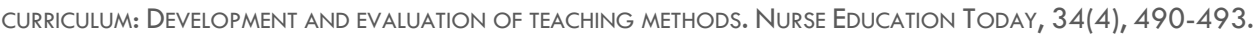

Richardson, .J, Grose, .J, Jackson, B., Gill, J., SAdeghian, H., BeCky, Hertel, J., et al. (2014A). Effect of Climate Change and resource SCARCity ON HEAlth CARE. NURSING StANDARD, 28(45), 44-9.

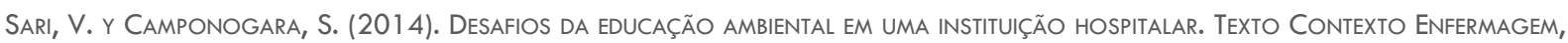
23(2), 469-478.

Sayre, L., Rhazl, N., Carpenter, H. y Hughes, N.l. (2010). Climate Change and human health: The role Of nuRSes in CONfronting the issue. NURSING AdMINISTRATION QUARTERLY, 34(4), 334-342.

Sendall, M., Lidstone, J., Fleming, M. y DOMOCol, M. (2013). NurSes and TeAchers: PARtnerships for Green Health Promotion. Journal OF SCHOOl HeAlth, 83(7), 508-513.

Stavrianopoulos, . T, Gourvelou, O. y Papadimitriou, M. (2010). The attitude Of nurses On Global Climate Change. InterscientifiC HeAlth CARE, 2(4), 155-162

Stavrianopoulos, T., Gourvelou, O. y GeVreki, E. (2010). The role of nursing science in global climate Change. To Vima tou ASKLIPIOU, 9(4), 460-472.

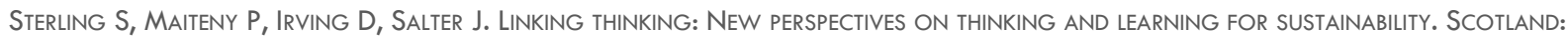
WORLD WILDLIFE FUND (WWF); 2005.

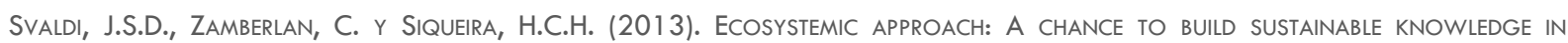
NURSING/HEALTH. EsCOla ANNA NeRY, 17(3), 542-547.

Thompson, T., Walpole, S., Braithwaite, I., InMAN, A., BARna, S. y MOrtimer, F. (2014). Learning objectives for sustainable health Care. THE LANCET, 384(9958), 1924-1925. 